\title{
Mobile Apps Developed by Government Organizations: A Boon for Fisheries and Aquaculture in India
}

\author{
S. Abbibrinda ${ }^{1 *}$, C. Llyod Chrispin ${ }^{2}$, R. Somu Sunder Lingam ${ }^{3}$ and S. Bharathi ${ }^{2}$ \\ ${ }^{1}$ Institute of Fisheries Biotechnology (IFBT), OMR campus, Vaniyanchavadi, Chennai, India \\ ${ }^{2}$ Dr. M.G.R Fisheries College and Research Institute, Ponneri, India \\ ${ }^{3}$ Krishnagiri-Barur Centre for Sustainable Aquaculture, Barur, Krishnagiri, India \\ *Corresponding author
}

\begin{abstract}
A B S T R A C T
Keywords

Smartphone, Applications, Play store, Fisheries, Aquaculture

Article Info

Accepted: 07 October 2020 Available Online: 10 November 2020

The foremost vital purpose of victimization of smartphones is for its applications which might be downloaded from the Play store. These mobile applications empower each capture fisheries and aquaculture. Using Mobile Application, Fishermen will ready to build the duty of fishing in a more pleasant way and, on the other side; a lot of profit may be taken from cultivation by diagnosing fish health diseases in time. Victimization of trendy technologies in fisheries and cultivation would be helpful to support higher cognitive process for coming up with and management of land use and alternative natural resources. The smartphone apps in Play store for fisheries and aquaculture created by Indian government organisations are mKRISHI ® Fisheries, Thoondil, FFMA (A Fisher Friend Mobile Application), Fish Disease Advisory, CIBA Shrimp app, INDAQUA, Treat My Fish, mJhinga For Shrimp Aquaculture, CIFT Lab Test, CIF Training, MathsyaKiran, Ornamental Fishes, Marine Fish Sales, SFDC App.
\end{abstract}

\section{Introduction}

Nowadays, mobile phones have become necessary for every person in their day today life. The usage of mobile applications has become essential in several fields. Smartphone apps were originally designed for productivity help like email, calendar, and phone databases, however, the general public demand for apps caused speedy enlargement into alternative areas such as mobile games, works automation, GPS and location-based services, order-tracking, and price ticket purchases. The demand of public leads to the creation of numerous apps in various fields. Applications are normally downloaded from application conveyance stages that are worked by the proprietor of the mobile OS just like the App Store (iOS) or Google Play Store ${ }^{(1,2)}$.

This paper explains about the use and importance of smartphone mobile applications in the field of fisheries and aquaculture. Aquaculture is the one of the leading revenue generating industry. Current fisheries production of India $(2018-19)$ is 13.42 million metric tonnes ${ }^{(3)}$. Now the government is launched a scheme PMMSY 
(Pradhan Mantri Matsya Sampada Yojana) for improving the fisheries and aquaculture sector for future. For this scheme, government has allotted Rs. 20,050crores. For achieving this, better awareness is needed among the farmers and fishermen's. This digital platform will help in achieving the sustainable and profitable aquaculture and fisheries production.

\section{Use of apps in fisheries and aquaculture}

The fishing community is one of the most vulnerable groups, facing a number of challenges threatening their lives and livelihoods like unpredictable weather and ocean states, danger zones in the sea, lack of GPS information on the fish shoal, quality fish processing, value addition and storage, market trends and government schemes. Mobile phones reached great heights in markets because it provides information in real time. By using Mobile Application, ocean Fishermen can do their work easily and safely as they get timely updates.

\section{Smartphone apps launched by Indian government organizations}

\section{mKRISHI® Fisheries}

It is a popular fisheries mobile app "mKRISHI®Fisheries" developed to provide information which help fishermen to decrease their operation cost. It is developed in collaboration with Tata Consultancy Services (TCS) Innovation Lab, Mumbai and INCOIS, Hyderabad and ICAR-CMFRI. This app makes fishing activities less expensive ${ }^{(4)}$. It gives advisories in local languages. It is a result of multi-dimensional analysis. It also provides data about sea surface temperature and the presence of phytoplankton. It is helpful to identify the regions which are unsafe in the ocean by the fishermen using the previous data of wind speed and wind direction. So it is advisable for the fishermen to go to the ocean only when the information map is blue in color ${ }^{(5)}$. Reduction in diesel consumption conjointly ends up in a less impure atmosphere. The other major challenge is lack of information signal accessibility in Deep Ocean. To increase the mobile signal up to thirty kilometers within the deep ocean, TCS and other partners made a pilot. This change the worth negotiation for the fishermen.

\section{Thoondil}

THOONDIL app is developed by Tamil Nadu Department of Fisheries jointly with National Centre for Coastal Research which provides real time data like weather forecast and potential fishing zone etc. It is a good support tool for saving numerable lives of fishermen. It is an android application launched to help fisherman community. This application compasses to fishing towns from Pulicat in Coromandal Coast to Neerody in West Coast (7). It has several vital options like a compass, weather, PFZ, daily journeys, my crew, my boats, live view, past trips, rescue set up, report incident and SOS. The live position of the boat can be found by its owner from the land by using live read feature in this app. In case of storm or cyclone, this app will be more useful to locate the boat and rescue the fishermen. This app reaches about 1076 Kmright along the whole Tamil Nadu.

\section{FFMA (A Fisher Friend Mobile Application)}

The app which was piloted by the MS Swaminathan Research Foundation (MSSRF) in partnership with Qualcomm and TCS in 2013. Everything, from Tsunamis to wave forecasts, is traced by INCOIS. MSSRF then uploads captured satellite pictures by INCOIS in this app successfully. All the information is delivered in 9 languages. It updates the data 
like fishing shoals movement and also marks danger zones like Sunken ship wreckages once in every 24 hours And also it updates additional data like wave height, wind speed once in every 6 hours. The Fisher Friend Mobile Application provides inputs to the fisher folk such as Potential Fishing Zone and TUNA species-specific forecast, GPS facility and traditional fishing zones. It provides disaster alerts such as cyclone, tsunami.

Other uses like marking of danger zones in the sea such as sunken boat, rock substrate, dead coral reefs and market prices for various fish varieties, International Border Line (IBL) alert with state, SOS (Save our Soul) possibility for rescue once within the essential scenario in the ocean, tracking fishing route, career facility together with crucial contact details. Additionally, it provides ocean state forecast information such as wind speed and direction, sea surface temperature, wave height, etc., and also the updates of government schemes and daily news (8). Around ten thousand fishermen, families are getting benefitted by this app.

\section{Fish disease advisory}

Fish Disease Advisory is an education app developed by ICAR-CIFRI (Central Inland Fisheries Research Institute). It was released on October 22, 2018. It was launched to provide information about fish diseases in inland open waters such as fungal, bacterial, viral, parasitic, nutritional, genetic deformities and prawn diseases. It also provides the disease gallery for those above mentioned diseases. As disease cause a major impact on fish production, in both capture and culture system, it is better to use this application to gain some knowledge about the diseases like its type, pathogen responsible, illness outbreaks, etc., in the real - time ${ }^{(9)}$. FDA is also useful for performing chemotherapy to battle against the disease.

\section{CIBA shrimp}

ICAR-Central Institute of Brackish water Aquaculture (CIBA), Chennai launched Vanami shrimp app. The farm management practices of Penaeus vannamei (Pacific white shrimp) is provided by this app for the shrimp farmers in a best way ${ }^{(10)}$. Users can post their queries. The answers for the queries will be posted within two working days. CIBA Shrimp app provides technical support to the shrimp farmers. It can be downloaded at free of cost in Google Play store ${ }^{(11)}$.

The updated version of this application contains risk assessment module, advisories, government regulations and disease diagnosis. It can also work without internet connection i.e., Offline.

\section{INDAQUA}

This app is developed by ICAR - Central Institute of Freshwater Aquaculture (CIFA), Bhubaneswar. It provides information indifferent local languages. It has many options like aqua directory, aqua expert, my farm, an aqua library, e-forum and recent updates. We can also use it as a portal in the web version. Information to the stakeholders in India can be provided with the help of this application $^{(11)}$.

\section{Treat my fish}

ICAR - Central Institute of Freshwater Aquaculture, Bhubaneswar launched a mobile application named "Treat My Fish", which is available in three languages like English, Hindi and Oriya. It is a farmer-friendly ICT tool. It provides advisories for freshwater fish health issues. It is available in play store at free of cost ${ }^{(12)}$. It contains information about parasitic, bacterial, fungal, viral and other diseases. This app can also be used for remote diagnosis. 


\section{mJhinga for aquaculture}

To provide detailed advisory to farmers on setting up of new ponds, mJhinga mobile application was launched by ICAR-CIFE, Central Institute of Fisheries Education, Mumbai with a funding support from the National Agricultural Higher Education Project. It was mainly developed for shrimp aquaculture. It shows current market price trends. This bilingual dynamic application is useful for farmers to identify the problems by themselves.. It also provides an option to ask the experts. There is a digital notebook to record and track daily inputs, expenses and water quality. This app is a great benefit for the farmers to empower inland saline aquaculture ${ }^{(13)}$. In this, we can also find a helpline number for technical and government support.

\section{CIFT lab test}

ICAR - Central Institute Fisheries Technology, Kochi launched "CIFT Lab Test" app in 2019. It provides information about microbiological parameters and quality parameters of water and ice samples ${ }^{(14)}$. Lab testing categories including analysis of various types of fish and fish products, packaging materials and other related parameters.

\section{CIFT training}

To provide full detailed information on ICAR-CIFT training programs, ICAR-Central Institute of Fisheries Technology, Cochin has developed this application which is very useful for the fisheries students and researchers ${ }^{(15)}$. It can be accessed at any time and registration for these training programs can be done through online mode. Industry personnel and state extension personnel are profited by this app.

\section{Mathsya Kiran}

Mathsya Kiran is a potential app which provides fisheries - related data on culture and management practices as text and attractive image format ${ }^{(16)}$. It is developed under a Masters Research program at ICAR-CIFE, Mumbai. It can be downloaded from the play store at free of cost.

It is very useful to fish farmers, students, extension personnel, policymakers. It can be accessed in offline mode also; thus it is userfriendly so anyone interested in fish farming can use this application easily.

\section{Ornamental fishes}

Ornamental fish rearing is a great hobby for everyone from old people to kids. It is majorly for aesthetic purposes. This app provides information for ornamental fish farming like lifespan, scientific name and temperature, etc.

It was developed by Tamil Nadu Dr J. Jayalalithaa Fisheries University ${ }^{(17)}$. It conjointly provides knowledge regarding the actual tank size of various decorative fishes and tank region specifically for every species.

\section{Marine fish scales}

Marine Fish Sales app is developed to provide a platform to facilitate direct sales between fisher folk and the customers. It is a multivendor e-commerce app which was launched under the NICRA project of ICAR-CMFRI (18)

The vendors are coastal fisher folk groups from whom customers can get fresh fish directly. It includes Payment options like cash on Delivery, net Banking, debit/credit cards to make purchasing easy for all types of consumers. 
Table.1 Mobile apps launched by Indian government organizations with its special features

\begin{tabular}{|c|c|c|c|c|c|c|c|c|}
\hline Sl.No & $\begin{array}{l}\text { Name of the } \\
\text { smartphone } \\
\text { Application }\end{array}$ & $\begin{array}{l}\text { Launched by (Indian } \\
\text { Government } \\
\text { Organizations) }\end{array}$ & $\begin{array}{l}\text { Size in } \\
\text { Megabytes } \\
\text { (Mb) }\end{array}$ & $\begin{array}{l}\text { Released } \\
\text { date }\end{array}$ & Updated date & $\begin{array}{l}\text { Latest } \\
\text { Version }\end{array}$ & $\begin{array}{l}\text { No. of } \\
\text { downloads }\end{array}$ & Special features \\
\hline 1. & mKRISHI®Fisheries & ICAR - CMFRI, TCS & 3.35 & 8 Mar 2017 & 22 May 2020 & 2.9 & $5,000+$ & $\begin{array}{l}\text { Increase the catch; reduce the cost of operations, } \\
\text { environment friendly. }\end{array}$ \\
\hline 2. & Thoondil & $\begin{array}{l}\text { Department of fisheries, } \\
\text { Tamil Nadu }\end{array}$ & 4.44 & $\begin{array}{ll}18 & \text { May } \\
2019 & \end{array}$ & 15 Nov 2019 & 2.0 & $10,000+$ & $\begin{array}{l}\text { Information about weather forecast and PFZ; } \\
\text { Cyclone alerts are close provided. }\end{array}$ \\
\hline 3. & FFMA & $\begin{array}{lr}\text { M.S } & \text { Swaminathan } \\
\text { Research } & \text { Foundation } \\
\text { (MSSRF) } & \\
\end{array}$ & 6.21 & $\begin{array}{ll}27 & \text { Dec } \\
2016 & \end{array}$ & 5 Jun 2020 & 4.63 & $10,000+$ & $\begin{array}{l}\text { PFZ, GPS facility; it also provides ocean state } \\
\text { forecast information and also disaster alerts. }\end{array}$ \\
\hline 4. & Fish Disease Advisory & ICAR - CIFRI & 5.23 & $\begin{array}{ll}22 & \text { Oct } \\
2018\end{array}$ & 22 Oct 2018 & 1.0 & $5,000+$ & $\begin{array}{l}\text { Fish diseases in inland open waters such as } \\
\text { fungal, bacterial, viral, parasitic, nutritional, } \\
\text { genetic deformities and prawn diseases. }\end{array}$ \\
\hline 5. & Vanami shrimp App & ICAR - CIBA & 25.03 & 4 Apr 2017 & 8 mar 2020 & 2.0 .4 & $10,000+$ & $\begin{array}{l}\text { Input calculators, BMP module, disease } \\
\text { diagnosis shrimp farm risk assessment module, } \\
\text { update and advisories, government regulations }\end{array}$ \\
\hline 6. & INDAQUA & ICAR - CIFA & 12.30 & 5 mar 2019 & 13 Nov 2019 & 2.7 & $1,000+$ & $\begin{array}{l}\text { Aqua directory, aqua expert, my farm, aqua } \\
\text { library, e-forum and recent updates }\end{array}$ \\
\hline 7. & Treat my Fish & ICAR - CIFA & 10.40 & 4 Dec 2018 & 20 Sep 2019 & 7.0 .1 & $1,000+$ & $\begin{array}{l}\text { Information about parasitic, bacterial, fungal, } \\
\text { viral and other diseases. }\end{array}$ \\
\hline 8. & $\begin{array}{l}\text { mJhinga for Shrimp } \\
\text { Aquaculture }\end{array}$ & ICAR - CIFE & 19.01 & $\begin{array}{ll}28 & \text { Sep } \\
2019 & \end{array}$ & 3 Dec 2019 & 0.0 .2 & $500+$ & $\begin{array}{l}\text { Shows current market price trends. For setting } \\
\text { up new ponds, it provides advisories to the } \\
\text { farmers. }\end{array}$ \\
\hline 9. & CIFT Lab test & $\begin{array}{l}\text { V. Chandrasekar ICAR - } \\
\text { CIFT }\end{array}$ & 7.45 & 6 Jun 2019 & 2 Dec 2019 & 3.0 & $100+$ & $\begin{array}{l}\text { Information about microbiological and quality } \\
\text { parameters of water and ice samples, analysis of } \\
\text { various types of fish and fish products, } \\
\text { packaging materials. }\end{array}$ \\
\hline 10. & CIFT training & $\begin{array}{l}\text { V. Chandrasekar } \\
\text { ICAR - CIFT }\end{array}$ & 7.86 & $\begin{array}{ll}7 & \text { June } \\
2019 & \end{array}$ & 3 Dec 2019 & 1.0 & $100+$ & $\begin{array}{l}\text { Provides information on ICAR - CIFT Training } \\
\text { programs. }\end{array}$ \\
\hline 11. & Mathsyakiran & $\begin{array}{l}\text { KiranmayiDhenavakonda } \\
\text { ICAR - CIFE }\end{array}$ & 26.32 & $\begin{array}{ll}11 & \text { Nov } \\
2019 & \end{array}$ & 11 Nov 2019 & 1.0 & $100+$ & $\begin{array}{l}\text { Provides fisheries related data on culture and } \\
\text { management practices as text and attractive } \\
\text { image format. }\end{array}$ \\
\hline 12. & Ornamental Fishes & TNJFU & 9.51 & 3 Oct 2018 & 3 Oct 2018 & 2.0 & $1,000+$ & $\begin{array}{l}\text { Provides information like size, lifespan, } \\
\text { breeding, scientific name, temperature and } \\
\text { feeding. }\end{array}$ \\
\hline 13. & Marine fisheries & $\begin{array}{l}\text { ICAR - CMFRI, } \\
\text { Zacharia PU }\end{array}$ & 2.03 & 1 Feb 2019 & 1 Feb 2019 & 1.0 & $100+$ & $\begin{array}{l}\text { To facilitate direct sales between fisher folk and } \\
\text { the customers }\end{array}$ \\
\hline 14. & SFDC App & $\begin{array}{l}\text { Department of Fisheries, } \\
\text { West Bengal }\end{array}$ & 10.23 & $\begin{array}{ll}27 & \text { Nov } \\
2019 & \end{array}$ & 12 Jun 2020 & 1.0.18 & $50,000+$ & Ecotourism, food court and online fish order. \\
\hline
\end{tabular}


Table.2 Similar apps developed by private agencies in the corresponding fields

\begin{tabular}{|c|c|c|c|c|c|c|c|c|c|}
\hline Sl.No & $\begin{array}{l}\text { Name of the } \\
\text { app }\end{array}$ & $\begin{array}{l}\text { Developer (Other } \\
\text { than Indian } \\
\text { government } \\
\text { organization) }\end{array}$ & $\begin{array}{l}\text { Category of the } \\
\text { app }\end{array}$ & $\begin{array}{l}\text { Size in } \\
\text { megabytes } \\
\text { (Mb) }\end{array}$ & Released date & Updated Date & Latest version & $\begin{array}{l}\text { Ratings (out } \\
\text { of } 5 \text { star) }\end{array}$ & $\begin{array}{l}\text { No.of } \\
\text { downloads }\end{array}$ \\
\hline 1. & Aqua Brahma & $\begin{array}{l}\text { Mile deep works Pvt. } \\
\text { Ltd. }\end{array}$ & Aquaculture & 5.57 & 21 Feb 2015 & 30 Oct 2018 & 2.6 & 4.3 & $50,000+$ \\
\hline 2. & $\begin{array}{l}\text { Aqua farmer } \\
\text { App }\end{array}$ & Aqua app & Aquaculture & 22.63 & 12 Feb 2018 & 12 Feb 2018 & 1.0 & 2.6 & $1,000+$ \\
\hline 3. & $\begin{array}{l}\text { Aquall app } \\
\text { marketing }\end{array}$ & $\begin{array}{l}\text { Aquall foods and } \\
\text { products Pvt.ltd }\end{array}$ & Aquaculture & 9.00 & 9 Aug 2016 & 11 Dec 2019 & 2.1 .11 & 4.3 & $10,000+$ \\
\hline 4. & Fish names & Leh & Aquaculture & 12.77 & 25 Sep 2016 & 9 Aug 2019 & 3.4 & 3.0 & $5,000+$ \\
\hline 5. & Pescare & Budding Developers & Aquaculture & 1.59 & 8 Nov 2017 & 14 Nov 2017 & 1.0 .1 & 4.9 & $100+$ \\
\hline 6. & INCOIS & INCOIS & Marine fisheries & 3.19 & 15 Feb 2016 & 15 Feb 2016 & 1.0 & 3.3 & $1,000+$ \\
\hline 7. & Machli & $\begin{array}{l}\text { Reliance Foundation } \\
\text { Information Services }\end{array}$ & Marine fisheries & 8.59 & 5 Aug 2019 & 2 Dec 2019 & 2.0 .11 & 4.0 & $50,000+$ \\
\hline 8. & Odaku & $\begin{array}{l}\text { Odaku online services } \\
\text { Pvt.Ltd }\end{array}$ & Marine Fisheries & 9.95 & 1 Nov 2015 & - & 64.0 & 4.8 & $1,000+$ \\
\hline 9. & $\begin{array}{l}\text { PFZ - } \\
\text { Advisory }\end{array}$ & Mobile seva & Marine fisheries & 1.35 & 9 Sep 2015 & 9 Sep 2015 & 1.0 & 3.7 & $500+$ \\
\hline 10. & $\begin{array}{l}\text { SARAT } \\
\text { (Search and } \\
\text { Rescue Aid } \\
\text { Tool) }\end{array}$ & INCOIS & Marine Fisheries & 5.73 & 14 Jul 2017 & 7 Oct 2018 & 1.3 .1 & 4.4 & $1,000+$ \\
\hline 11. & Sagara & NIC eGov mobile apps & Marine fisheries & 3.24 & 25 feb 2018 & 30 May 2018 & 2.0 & 4.4 & $5,000+$ \\
\hline 12. & Aqua Pulse & Pinnacle Soft & marketing & 4.49 & 1 Aug 2017 & 26 Sep 2017 & 2.0 & 4.2 & $100+$ \\
\hline 13. & $\begin{array}{l}\text { Daily fish } \\
\text { India }\end{array}$ & $\begin{array}{l}\text { Baby marine } \\
\text { Enterprise }\end{array}$ & Marketing & 8.57 & 9 Feb 2016 & - & 2.0 .024 & 2.8 & $1,00,000+$ \\
\hline 14. & Healthy Fish & Healthy Fish & Marketing & 5.09 & 6 Dec 2016 & 16 Mar 2020 & 6.7 & 4.2 & $5,000+$ \\
\hline 15. & $\begin{array}{l}\text { Matha Fresh } \\
\text { Fish }\end{array}$ & Matha Fresh Fish & Marketing & 3.57 & 29 Mar 2018 & 23 mar 2020 & 1.0 .2 & 4.2 & $10,000+$ \\
\hline 16. & $\begin{array}{l}\text { Nallamee.co } \\
\mathrm{m}\end{array}$ & $\begin{array}{l}\text { Infametech Solutions } \\
\text { Pvt.Ltd }\end{array}$ & Marketing & 6.65 & 28 Oct 2017 & 7 Oct 2017 & 1.0 .7 & 3.6 & $5,000+$ \\
\hline 17. & $\begin{array}{l}\text { Fresh Fish } \\
\text { Cart }\end{array}$ & Astin Soft Pvt. Ltd & Marketing & 5.38 & 14 Jun 2017 & 5 Jul 2019 & 6.5 & 4.3 & $5,000+$ \\
\hline 18. & Aqua Deals & $\begin{array}{l}\text { Mile deep works Pvt. } \\
\text { Ltd }\end{array}$ & Marketing & 4.91 & 12 Feb 2017 & 03 Mar 2020 & 4.1 & 4.2 & $10,000+$ \\
\hline
\end{tabular}

Common features of the apps under a particular category and special features in these government's apps 
Table.3 Comparison of the apps developed by Indian government organizations with the similar apps launched by other private agencies

\begin{tabular}{|c|c|c|c|c|c|c|c|}
\hline Sl.No & $\begin{array}{l}\text { Name of the apps } \\
\text { launched by } \\
\text { Indian government } \\
\text { organizations }\end{array}$ & $\begin{array}{l}\text { Categories under } \\
\text { which the Indian } \\
\text { government } \\
\text { organizations apps are } \\
\text { included }\end{array}$ & $\begin{array}{l}\text { Similar apps in the } \\
\text { same category } \\
\text { launched by other } \\
\text { private agencies }\end{array}$ & $\begin{array}{l}\text { Common features of the specific } \\
\text { category apps }\end{array}$ & $\begin{array}{l}\text { Special features of the } \\
\text { apps developed by } \\
\text { Indian government } \\
\text { organizations }\end{array}$ & $\begin{array}{l}\text { Total } \\
\text { number of } \\
\text { apps in the } \\
\text { same field }\end{array}$ & $\begin{array}{l}\text { Percentage } \\
\text { (Categories) }\end{array}$ \\
\hline 1. & $\begin{array}{l}\text { mKRISHI® } \\
\text { Fisheries }\end{array}$ & Marine Fisheries & $\begin{array}{l}\text { INCOIS, Machi, Odaku, } \\
\text { PFZ - Advisory, } \\
\text { SARAT (Search and } \\
\text { Rescue Aid Tool), } \\
\text { Sagara }\end{array}$ & $\begin{array}{l}\text { Provide information about the } \\
\text { potential fishing zone, wind speed, } \\
\text { direction, wave heights, GPS, } \\
\text { Tsunami alerts and ocean State } \\
\text { forecast }\end{array}$ & $\begin{array}{l}\text { Increase the catch; reduce } \\
\text { the cost of operation, } \\
\text { environment friendly. }\end{array}$ & 9 & 30.00 \\
\hline 2. & Thoondil & Marine Fisheries & $\begin{array}{l}\text { INCOIS, Machi, Odaku, } \\
\text { PFZ - Advisory, } \\
\text { SARAT (Search and } \\
\text { Rescue Aid Tool), } \\
\text { Sagara }\end{array}$ & $\begin{array}{l}\text { Provide information about the } \\
\text { potential fishing zone, wind speed, } \\
\text { direction, wave heights, GPS, } \\
\text { tsunami alerts and ocean state } \\
\text { forecast }\end{array}$ & $\begin{array}{l}\text { Information about } \\
\text { weather forecast and PFZ; } \\
\text { Cyclone alerts also } \\
\text { provided }\end{array}$ & 9 & 30.00 \\
\hline 3. & $\begin{array}{l}\text { FFMA ( A Fisher } \\
\text { Friend Mobile } \\
\text { Application) }\end{array}$ & Marine Fisheries & $\begin{array}{l}\text { INCOIS, Machi, Odaku, } \\
\text { PFZ - Advisory, } \\
\text { SARAT (Search and } \\
\text { Rescue Aid Tool), } \\
\text { Sagara }\end{array}$ & $\begin{array}{l}\text { Provide information about the } \\
\text { potential fishing zone, wind speed, } \\
\text { direction, wave heights, GPS, } \\
\text { Tsunami alerts and ocean State } \\
\text { forecast }\end{array}$ & $\begin{array}{l}\text { PFZ, GPS facility; it also } \\
\text { provides ocean state } \\
\text { forecast information and } \\
\text { also disaster alerts }\end{array}$ & 9 & 30.00 \\
\hline 4. & $\begin{array}{l}\text { Fish Disease } \\
\text { Advisory }\end{array}$ & Aquaculture & $\begin{array}{l}\text { Aqua Brahma, Aqua } \\
\text { farmer App, Aquall fish } \\
\text { names, Pescare }\end{array}$ & $\begin{array}{l}\text { Daily news updates about this field, } \\
\text { latest market prices, videos and } \\
\text { events related to aquaculture, } \\
\text { database of stakeholders, FAQ and } \\
\text { discussion forums }\end{array}$ & $\begin{array}{l}\text { Fish diseases in inland } \\
\text { open waters such as } \\
\text { fungal, bacterial, viral, } \\
\text { parasitic, genetic } \\
\text { deformities and prawn } \\
\text { diseases }\end{array}$ & 12 & 40.00 \\
\hline 5. & CIBA shrimp app & Aquaculture & $\begin{array}{l}\text { Aqua Brahma, Aqua } \\
\text { farmer App, Aquall fish } \\
\text { names, Pescare }\end{array}$ & $\begin{array}{l}\text { Daily news updates about this field, } \\
\text { latest market prices, videos and } \\
\text { events related to aquaculture, } \\
\text { database of stakeholders, FAQ and } \\
\text { discussion forums }\end{array}$ & $\begin{array}{l}\text { Input calculators, BMP } \\
\text { module, disease diagnosis } \\
\text { shrimp farm risk } \\
\text { assessment module, } \\
\text { update and advisories, } \\
\text { government regulations }\end{array}$ & 12 & 40.00 \\
\hline 6. & INDAQUA & Aquaculture & $\begin{array}{l}\text { Aqua Brahma, Aqua } \\
\text { farmer App, Aquall fish } \\
\text { names, Pescare }\end{array}$ & $\begin{array}{l}\text { Daily news updates about this field, } \\
\text { latest market prices, videos and } \\
\text { events related to aquaculture, } \\
\text { database of stakeholders, FAQ and } \\
\text { discussion forums }\end{array}$ & $\begin{array}{l}\text { Aqua directory, aqua } \\
\text { expert, my farm, aqua } \\
\text { library, e-forum and } \\
\text { recent updates }\end{array}$ & 12 & 40.00 \\
\hline 7. & Treat my fish & Aquaculture & $\begin{array}{l}\text { Aqua Brahma, Aqua } \\
\text { farmer App, Aquall fish } \\
\text { names, Pescare }\end{array}$ & $\begin{array}{l}\text { Daily news updates about this field, } \\
\text { latest market prices, videos and } \\
\text { events related to aquaculture, }\end{array}$ & $\begin{array}{l}\text { Information about } \\
\text { parasitic, bacterial, } \\
\text { fungal, viral and other }\end{array}$ & 12 & 40.00 \\
\hline
\end{tabular}




\begin{tabular}{|c|c|c|c|c|c|c|c|}
\hline & & & & $\begin{array}{l}\text { database of stakeholders, FAQ and } \\
\text { discussion forums. }\end{array}$ & diseases & & \\
\hline 8. & $\begin{array}{l}\text { mJhinga for shrimp } \\
\text { aquaculture }\end{array}$ & Aquaculture & $\begin{array}{l}\text { Aqua Brahma, Aqua } \\
\text { farmer App, Aquall fish } \\
\text { names, Pescare }\end{array}$ & $\begin{array}{l}\text { Daily news updates about this field, } \\
\text { latest market prices, videos and } \\
\text { events related to aquaculture, } \\
\text { database of stakeholders, FAQ and } \\
\text { discussion forums. }\end{array}$ & $\begin{array}{l}\text { Shows current market } \\
\text { price trends. For setting } \\
\text { up new ponds, it provides } \\
\text { advisors to the farmers. }\end{array}$ & 12 & 40.00 \\
\hline 9. & CIFT lab test & Aquaculture & $\begin{array}{l}\text { Aqua Brahma, Aqua } \\
\text { farmer App, Aquall fish } \\
\text { names, Pescare }\end{array}$ & $\begin{array}{l}\text { Daily news updates about this field, } \\
\text { latest market prices, videos and } \\
\text { events related to aquaculture, } \\
\text { database of stakeholders, FAQ and } \\
\text { discussion forums. }\end{array}$ & $\begin{array}{l}\text { Information about } \\
\text { microbiological and } \\
\text { quality parameters of } \\
\text { waters and ice samples }\end{array}$ & 12 & 40.00 \\
\hline 10. & CIFT training & Aquaculture & $\begin{array}{l}\text { Aqua Brahma, Aqua } \\
\text { farmer App, Aquall fish } \\
\text { names, Pescare }\end{array}$ & $\begin{array}{l}\text { Daily news updates about this field, } \\
\text { latest market prices, videos and } \\
\text { events related to aquaculture, } \\
\text { database of stakeholders, FAQ and } \\
\text { discussion forums. }\end{array}$ & $\begin{array}{l}\text { Provides information on } \\
\text { ICAR - CIFT training } \\
\text { programs }\end{array}$ & 12 & 40.00 \\
\hline 11. & MathsyaKiran & Aquaculture & $\begin{array}{l}\text { Aqua Brahma, Aqua } \\
\text { farmer App, Aquall fish } \\
\text { names, Pescare }\end{array}$ & $\begin{array}{l}\text { Daily news updates about this field, } \\
\text { latest market prices, videos and } \\
\text { events related to aquaculture, } \\
\text { database of stakeholders, FAQ and } \\
\text { discussion forums. }\end{array}$ & $\begin{array}{l}\text { Provides fisheries related } \\
\text { data on culture and } \\
\text { management practices as } \\
\text { text and attractive image } \\
\text { format. }\end{array}$ & 12 & 40.00 \\
\hline 12. & Ornamental fishes & Aquaculture & $\begin{array}{l}\text { Aqua Brahma, Aqua } \\
\text { farmer App, Aquall fish } \\
\text { names, Pescare }\end{array}$ & $\begin{array}{l}\text { Daily news updates about this field, } \\
\text { latest market prices, videos and } \\
\text { events related to aquaculture, } \\
\text { database of stakeholders, FAQ and } \\
\text { discussion forums. }\end{array}$ & $\begin{array}{l}\text { Provides information like } \\
\text { size, lifespan, breeding, } \\
\text { scientific name, } \\
\text { temperature and feeding }\end{array}$ & 12 & 40.00 \\
\hline 13. & Marine Fish sales & Marketing & $\begin{array}{l}\text { Aqua pulse, Daily fish } \\
\text { India, Healthy fish, } \\
\text { Matha fresh fish, } \\
\text { Nallameen.com, fresh } \\
\text { fish cart, Aqua Deals }\end{array}$ & $\begin{array}{l}\text { Direct sales between fisher folk and } \\
\text { customers, delivery, sells feed, } \\
\text { machine health care products,etc. }\end{array}$ & $\begin{array}{l}\text { To facilitate direct sales } \\
\text { between fisher folk and } \\
\text { the customers }\end{array}$ & 9 & 30.00 \\
\hline 14. & SFDC app & Marketing & $\begin{array}{l}\text { Aqua pulse, Daily fish } \\
\text { India, Healthy fish, } \\
\text { Matha fresh fish, } \\
\text { Nallameen.com, fresh } \\
\text { fish cart, Aqua Deals }\end{array}$ & $\begin{array}{l}\text { Direct sales between fisher folk and } \\
\text { customers, delivery, sells feed, } \\
\text { machine health care products etc. }\end{array}$ & $\begin{array}{l}\text { Ecotourism food court } \\
\text { and online fish order }\end{array}$ & 9 & 30.00 \\
\hline
\end{tabular}


Fig.1 Name and logo of the smartphone apps for fisheries and aquaculture created by Indian government organizations

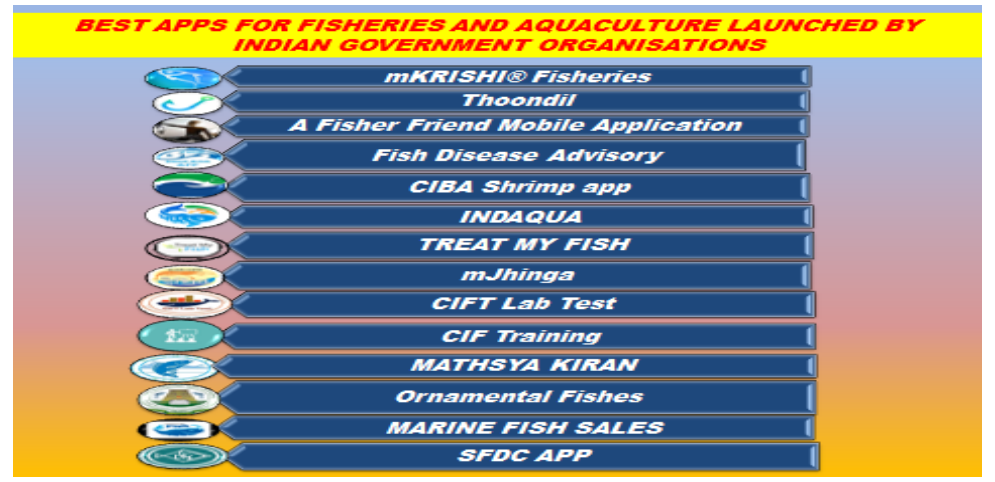

Fig.2 Comparison of these apps in number of downloads

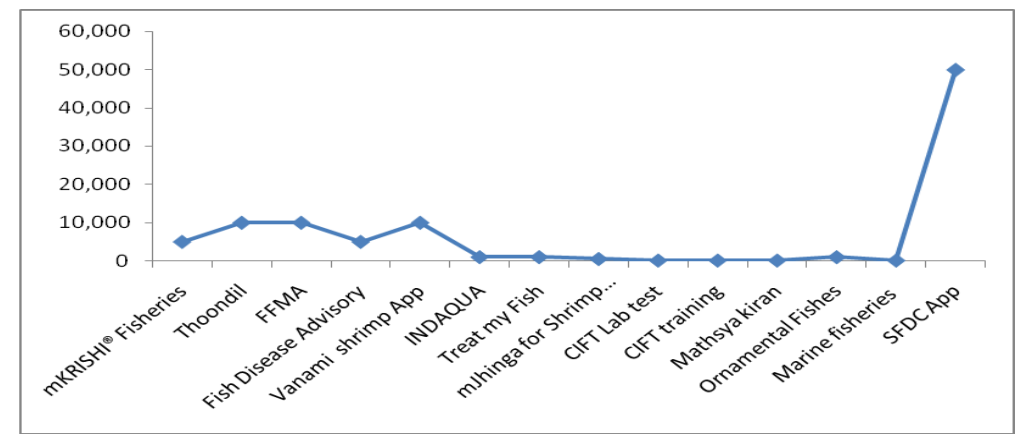

Fig.3 Percentage of total number of apps launched by Indian government organizations in particular categories

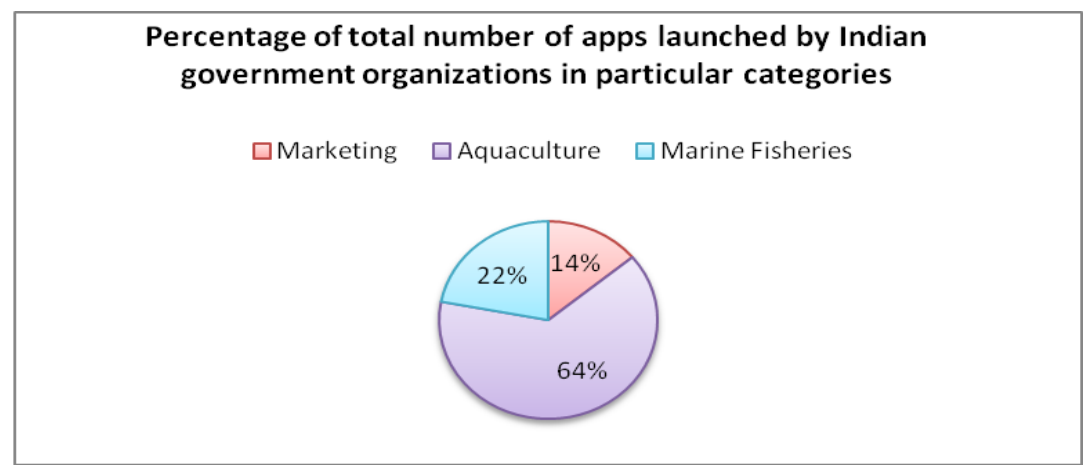

\section{SFDC app}

SFDC App is an online fish delivery app developed by the State Department of Fisheries, West Bengal ${ }^{(19)}$. It has options like ecotourism, food court and online fish order. Eco tourism provides information about the list of hotels and types of rooms available. In the food court section, under food categories; lunch and dinner, snacks and starters are available. Dressed fish, dried fish, fillet fish items and ready to fry items can also be ordered through online fish order option. 
Mobile applications with its combined features

Each smartphone application launched by Indian government organizations which are downloaded from the play store used in fisheries and aquaculture field has its own unique features and size. They are listed below:

In conclusion this modern world, usage of mobile application becomes mandatory in everyone's life. In developing countries, smart phone usage has become very popular. For prevention and precaution, the mobile applications are highly useful for the fisherman. Mobile application helps the fishermen to know the seller and buyer. In Indian scenario, there are more mobile applications have been developed but they have not reached the stakeholders, fishing community and fish farmers, due to language issues. However, there are some apps provide multilingual choice which is very useful to them. Thus, in future, the smartphone apps with user-friendly mode and provide much information in their local languages need to be developed for the welfare of fisherman and aqua culturist.

\section{References}

1. Zhang, D., and Adipat, B. (2005). Challenges, methodologies, and issues in the usability testing of mobile applications. International journal of human-computer interaction, 18(3), 293308.

2. Sarwar, M., and Soomro, T. R. (2013). Impact of smartphone's on society. European journal of scientific research, 98(2), 216-226.

3. National Fisheries Policy, National Fisheries Development Board, 2020.

4. Anon (2017, March 8) mKRISHI®Fisheries. Retrieved from https://play.google.com/store/apps/details ?id=com.tcs.fish.mkrishi\&hl=en_US

5. Anon (2017, January 19) New mobile app developed to help fishermen. NDTV. Retrieved

fromhttps://www.ndtv.com/indianews/new-mobile-app-developed-tohelp-fishermen-1650465

6. Government of Tamil Nadu Department of Fisheries (2019, May 18) Thoondil Fisherman Friendly App by TN Gov. \& NCCR. Retrieved from https://www.fisheries.tn.gov.in/Thoondil

7. Anon (2016, December 27) A FISHER FRIEND MOBILE APPLICATION (FFMA). Retrieved fromhttps://play.google.com/store/apps/d etails?id=com.mssrf.ffma\&hl=en_IN14.

8. Anon (2018, October 22) Fish Disease Advisory. Retrieved fromhttps://play.google.com/store/apps/d etails?id=pathak. . pratik.fishdiseaseadvisor $\mathrm{y} \& \mathrm{hl}=\mathrm{en} 19$.

9. Anon (2017, April 4) ICAR-CIBA launched "Vanami Shrimpapp" a mobile app on Pacific white shrimp (Penaeus vannamei) farming ICAR. Retrieved from https://icar.org.in/node/8248

10. Anon (2017, April 4) Vanami Shrimpapp. Retrieved fromhttps://play.google.com/store/apps/d etails?id=com.vanami $\cdot$.shrimpapp\&hl=en IN20.

11. Anon (2019, March 5) INDAQUA. Retrieved fromhttps://play.google.com/store/apps/d etails?id=com.indaquaicar\&hl=en23.

12. Anon (2018, December 4) Treat My Fish. Retrieved from https://play.google.com/ store/apps/details?id=com.webfarms.treat myfish\&hl=en_IN24.

13. Anon (2019, September 28) mJhinga for Shrimp Aquaculture. Retrieved fromhttps://play.google.com/store/apps/d etails?id=in.kultivate.icarcife\&hl=en_AU 14. Anon (2019, June 6) CIFT Lab Test. 
Retrieved from https://play.google.com/store/apps/details ?id=com.vcsecon.CIFT_Lab_Test\&hl=en IN27.

15. Anon (2019, June 7) CIFTraining. Retrieved from https://play.google.com/store/apps/details ?id=com.vcsecon.CIFT_Training_new\&h l=en_IN27.

16. Anon (2019, November 11) Mathsyakiran. Retrieved from https://play.google.com/store/apps/details ?id=com.cife.mathsyakiran.mathsyakiran \&hl=en_IN27.
17. Anon (2018, October 3) Ornamental Fishes. Retrieved from https://play.google.com/store/apps/details ?id=com.cofe.fish_profile \&hl=en_IN27.

18. Anon (2019, February 1) Marine Fish Sales. Retrieved from https://play.google.com/store/apps/details ?id=com.tech.android.marinefishsales \&hl $=$ en_IN27.

19. Anon (2019, November 27) SFDC App. Retrieved from https://play.google.com/store/apps/details ?id=com.app.wbsdfc\&hl=en_IN27.

\section{How to cite this article:}

Abbibrinda, S., C. Llyod Chrispin, R. Somu Sunder Lingam and Bharathi, S. 2020. Mobile Apps Developed by Government Organizations: A Boon for Fisheries and Aquaculture in India. Int.J.Curr.Microbiol.App.Sci. 9(11): 588-598.

doi: https://doi.org/10.20546/ijcmas.2020.911.072 